\title{
The Psychological Trauma in Children and Adolescents: Scientific and Sociological Profiles
}

\author{
Nicola Malizia \\ University of Enna “Kore”, Enna, Italy \\ Email: nicola.malizia@unikore.it
}

How to cite this paper: Malizia, N. (2017). The Psychological Trauma in Children and Adolescents: Scientific and Sociological Profiles. Sociology Mind, 7, 11-25.

http://dx.doi.org/10.4236/sm.2017.71002

Received: November 7, 2016

Accepted: January 9, 2017

Published: January 12, 2017

Copyright $\odot 2017$ by author and Scientific Research Publishing Inc. This work is licensed under the Creative Commons Attribution International License (CC BY 4.0).

http://creativecommons.org/licenses/by/4.0/

\begin{abstract}
Despite the research on trauma, which until recent times has been directed towards the category of adults, today scholars need to turn their attention to children and adolescents who may undergo traumatic experiences at different stages of their growth. There is a prevailing hypothesis that the narrow and partial cognitive processes of memory, attention and dysfunctional problem solving skills constitute the main problem of exposure to trauma, which also compromises the family equilibrium, taking into account that traumatic events are arbitrary and unpredictable. It is possible that dissociation phenomena and derealization emerge in response to a traumatic event. The specificity and complexity of the treatment of disorders resulting from traumatic development have prompted specialists of different orientations to formulate guidelines and treatment protocols to guide clinical work.
\end{abstract}

\section{Keywords}

Psychological Trauma, Children, Adolescents, Trauma in the Family,

Treatments for Trauma, Trauma and Society

\section{Introduction}

Psychological trauma is generally the direct personal experience of an event that may cause or lead to death or serious injury, or other threats to the physical integrity. In addition, the definition of psychological trauma must be extended to include relational aspects such as serious threats not to physical integrity of an organism but to the fabric of one's relationships. Psychological trauma is considered as being present at an event which involves death, injury, or a threat to the physical integrity of another person; or to become aware of the unexpected or violent death, serious harm, or threat of death or injury experienced by a family member or another person with whom you are closely related. The trauma does not have a salutary effect on children and adolescents.

Trauma is defined as an emotionally unsustainable event for those who suffer. Ab- 
andonment is traumatic, for example, when it occurs in childhood where the care provided by adults is basic and abandonment is a serious threat. Finally, trauma can seriously affect the stages of child development.

\section{The Child and the Stages of Development}

Child development normally proceeds in stages, each of which provides basic tasks including the acquisition of balanced emotion regulation and moral reasoning. If trauma occurs in certain stages of development there is a real danger that the newly acquired skills and capabilities could be placed in jeopardy.

a) Childhood and early dyadic relationships

In the past, it was thought that children were excluded from traumatic conditions, as they were unaware of the dangers. On the contrary, today, very young children respond to trauma and resultant suffering. During the first year of life it is not only neurobiological maturation which influences psychosocial processes, but also social experiences. In particular, interactions with caregivers change the structure of the brain and the functional organization and make the early family relationships for child development vital. In addition, recent studies have shown that children notice the fear of traumatized parents and memorize the family atmosphere. This involves elevated heart rate, blood pressure beyond normal levels, intense breathing and expression of psychological suffering through weeping and shouting.

b) Preschoolers

Bretherton argued that the symbolic processes appear to be important in recovery from trauma because children are at the peak of symbolic play and communicate with animals and fantasy figures. Moreover, these symbolic and metaphorical thoughts do not end during the period of childhood. They become dormant for a little time and can form a context of greater psychological experiences during adolescence and adulthood. There is evidence that exposure to trauma makes the symbolic processes particularly vulnerable in toddlers and preschoolers.

An important aspect of the interventions for traumatized children is the encouragement to draw their own painful memories and bring them to mind in symbolic games, metaphorical stories and fairy tales and revive their nightmares in therapeutic and safe conditions. Thus, there is the belief that the symbolic processes of children work as a kind of self-healing practice, yet the theoretical insights of this, as well as empirical evidence, are poor. Nightmares, night terrors and anxiety dreams are common among young children and are considered part of post-traumatic stress disorder. Nightmares include highly vivid and terrifying scenes, but are devoid of narrative features such as the story of the dream, the scenes, the characters, emotions and messages. The current understanding of the responses of children to trauma, however, mainly emphasizes the pathological elements of the games and of the dreams in traumatic conditions (Pynoos Steinberg, \& Piacentini, 1999). Regulation of emotions is an important task for children. They are encouraged to control their aggressive outbursts and are taught to respect other children and to take them into account. With complex verbal skills, they are capable of negotiating in case of conflicts and are aware of their needs, as well as the different motivations and reasoning of other people. Physical attacks usually diminish during mid- 
dle childhood (Tremblay, 2000) but in life-threatening environments, as in the case of war, children may be faced with difficulties in controlling their aggressive impulses.

c) Childhood and puberty

At puberty self-esteem is linked to the acceptance of peers, from good friendships to success in school performance. Interactions with friends and peers have many of the functions that the family had before such as companionship, sharing, motivation and support (O'Brien \& Bierman, 1988). Today, it is well-known that a severe trauma may interfere with the delicate transition from childhood to adolescence and that attachment relationships in childhood are critical for the sense of security and as a safe haven to cope with fear. The attachment behavior is activated when life is endangered and in other traumatic situations. Therefore, to deal with the trauma, even older children rely on parents. They may fear constantly that something very unpleasant can happen to the members of their family and this concern for the security of the family can disrupt relationships with friends and peers. Cognitive and emotional skills became more complicated, flexible, integrated and disseminated during puberty. Traumatic experiences increase concentration and emotional problems, which in turn can harm the phase of development progress. Traumatized children may not be able to give their best performance, so they are unable to reach their maximum potential in schoolwork, which can lead to great frustration for themselves, parents and teachers alike.

d) Adolescence

Adolescence is considered a complex period because it includes significant changes in social relationships and in the responsibilities to society, which affect the brain and hormonal development. Teenagers establish their most important intimate relationships and shape their personality, their identity and their lifestyle. The effect of trauma in these areas can be profound and may lead to future plans shattered, and the risk of depression behaviors. Intrusive and uncontrollable memories of the traumatic scene can disturb intimate relationships and push teenagers to retreat from social contacts. Being the target of a trauma produced by man, as in the case of violence or rape, causes a profound lack of trust in the benevolence of the human race, which is often generalized in intimate relationships (Janoff-Bulman, 1997). There is no evidence to the effect that the trauma causes problems in identity formation of adolescents, including a vision of the world in extreme terms (van der Kolk, 1997) and the development of values (Baker \& Shallioub-Kevorkian, 1999).

\section{Trauma and Impact in Children and Adolescents}

The latest scientific researches on trauma have focused on school-age children and adolescents. Mental health problems such as PTSD and depressive symptoms, were the main focus, although little is known regarding the effect of trauma on cognitive development, nor social and emotional development of the child. There is a prevailing hypothesis that the narrow and partial cognitive processes of memory, attention and dysfunctional problem-solving skills are the main problems of exposure to trauma (Feeny, Foa, Treadwell, \& March, 2004). Empirical evidence confirms that children exposed to traumatic events show deficient school performance and suffer from concentration problems. It was found that abused children show few memories and often partial, in particu- 
lar those relating to the stories (Howe et al., 2004); moreover, they show attention disorders, generalized state of vigilance and awareness of the danger (Shields \& Cicchetti, 1998).

Thus, trauma means it is a vicious circle for the child's development since the school failures cause additional stress, providing an additional risk for mental health among traumatized children. Social support is regarded as one of the most important protective factors among adult survivors to trauma (Ozer et al, 2004). It is well-known, in particular in middle childhood that acceptance by peers and solitary friendships are fundamental to mental health, as well as a family atmosphere and good friendly relations which can serve as a protection against the negative effects of stress and trauma (Collishaw et al., 2007). Traumatic events appear to increase negative behaviors, as well as isolation and aggression and these behavioral patterns can decrease the necessary skills to maintain friendships and may lead to reduced popularity and greater loneliness (Hodges, Boivin, Vitaro, \& Bukowski, 1999).

Research in the clinical setting document that family trauma is associated with relationships between siblings is both problematic and protective. Leavitt, Gardner, Callagher, \& Schamess (1998) describe four models of relationship between brothers and sisters connected to trauma: 1) an absent fraternal relationship refers to detached and not meaningful relationships which are the result of a failure in the parent-child relationship; 2) the "locked adult model" implies relationships between closely related brothers and sisters who serve as a replacement for insecure or pathological relationships with parents; 3 ) in another model, the brothers collaborate to reactivate the difficulties they have experienced in the problematic relationship with parents; 4) in the "shield patter" of the trauma, a child adopts a protective role of the other brothers against the negative effects of trauma. The emotional processing such as recognizing the mind-body connection between fear responses and regulation of overwhelming feelings is an important therapeutic element in the treatment of traumatized children. Research on the emotional consequences of exposure to trauma has primarily focused on the psychopathology and has therefore overlooked a development approach and processing of emotions.

Childhood and adolescence involve dynamic developments in the way of expression, regulation, understanding and interpretation of emotions and recognition of same in themselves and in others. The ability to calm down and the management of painful feelings are considered extremely important for mental health in traumatic situations. It is thought that trauma has a negative impact on emotional development, such as the lowering of control or excessive control, expressed as overwhelming and uncontrollable feelings (Moradi et al., 1999). Traumatized children typically show their feelings in behavioral levels. Consequently, an impulsive behavior is common. Impulsiveness has a tendency to transform the feelings and tensions directly into action without cognitive assessments or processing emotions, which can allow an immediate escape from danger. Traumatic experiences in early and preverbal development and preverbal are encoded as a simple approach or avoid mnemonic patterns that can explain why procedural and emotional-kinesthetic expressions predominate among traumatized children. Optimal doses of accurate recognition of danger are important in child development. 
Traumatized children are, however, constantly in alarm. Empirical research on maltreated children showed an incorrect recognition of anger and fear and a limited expression of emotions (Maughan \& Cicchetti, 2002). In other words, these children are automatically tuned to recognize anger to protect themselves, so it is as if traumatized children could become experts in the recognition and discrimination of feelings of fear and anger signals and threats (Table 1).

\section{Trauma and Social Relations}

Trauma compromises family harmony and family equilibrium. Families are therefore unable to prepare themselves mentally and physically to handle trauma and parents can feel as if they themselves have no control. The children feel that their parents are not able to protect them and this fundamentally changes the family relationships and communication. Trauma then creates a specific family communication in which family members are saved by each other having full awareness of traumatic events. Parents may feel helpless when their children show symptoms and suffer the consequences of trauma. For example, if traumatized children show a decrease of interest in primitive important activities, such as games and friendship, parents should try to encourage them. However, in doing this, they are grappling with a strong conflict derived from the trauma and the fear of not succeeding. Changes in children's behavior, and even in personality, entail a huge burden for parents. The main task of parents is to protect their children from danger, from danger, threat and stress, because having a child who is victim of a trauma is so painful for adults. In many cases, such as traffic accidents, disasters and violence caused by war, both parents and children are affected.

It is generally agreed that a positive education, and a harmonious and sensitive mother-child interaction can improve the development of the baby's health in traumatic conditions. This in turn leads to a vicious circle in which the parents, who are themselves traumatized, make excessive demands and easily experience feelings of guilt and failure. Children, according to attachment theories, learn how to seek refuge, express emotions and push themselves and others towards the first parent-child interactions. Children are distinguished on the basis of their ability and motivation to explore the environment and rely on adults when they feel threatened. A relationship of secure attachment with significantly available adults provides a secure base for the child to explore the environment. Insecure attachment relationships, in turn, force children to seek protection elsewhere. The avoidant insecure children learn to believe in themselves and to deny their need while ambivalent insecure children cling to their parents or other adults, and they are overwhelmed by their fear of abandonment. Lacking there is a lack of systematic research on how attachment relationships develop in families exposed to traumatic events. The main task during the first year of life is to create a secure base in a close and reciprocal relationship with the caregiver. Almqvist \& Broberg (1997) have analyzed how traumatic events can cause problems in this task and finish in the worst case in a dyadic reactive attachment disorder. In a similar way, the available analyses show that adverse living conditions, dangers and threats test the child-caregiver dyads (Scheeringa \& Zeanah, 2001). Evidence shows that childhood trauma like sexual abuse and neglect and economic hardship represent a risk of insecure attachment. 
Table 1. Domains of impairment in children exposed to complex trauma.

\section{Attachment}

-Uncertainty about the reliability and predictability of the world

-Problems with boundaries

-Distrust and suspiciousness

-Social isolation

-Interpersonal difficulties

-Difficulty attuning to other people's emotional states

-Difficulty with perspective taking

-Difficulty enlisting other people as allies

\section{Biology}

-Sensorimotor developmental problems

-Hypersensitivity to physical contact

-Analgesia

-Problems with coordination, balance, body tone

-Difficulties localizing skin contact

-Somatization

-Increased medical problems across a wide span, e.g., pelvic pain, asthma, skin problems, autoimmune disorders, pseudo-seizures

\section{Affect Regulation}

-Difficulty with emotional self-regulation

-Difficulty describing feelings and internal experience

-Problems knowing and describing internal states

-Difficulty communicating wishes and desires

\section{Dissociation}

-Distinct alterations in states of consciousness

-Amnesia

-Depersonalization and derealization

-Two or more distinct states of consciousness, with impaired memory for state-based events

\section{Behavioral Control}

-Poor modulation of impulses

-Self-destructive behavior

-Aggression against others

-Pathological self-soothing behaviors

-Sleep disturbances

-Eating disorders

-Substance abuse

-Excessive compliance

-Oppositional behavior

-Difficulty understanding and complying with rules

-Communication of traumatic past by re-enactment in day-to-day behavior or play 


\section{Continued}

\section{Cognition}

-Difficulties in attention regulation and executive functioning

-Lack of sustained curiosity

-Problems with processing novel information

-Problems focusing on and completing tasks

-Problems with object constancy

-Difficulty planning and anticipating

-Problems understanding own contribution to what happens to them

-Learning difficulties

-Problems with language development

-Problems with orientation in time and space

-Acoustic and visual perceptual problems

-Impaired comprehension of complex visual-spatial patterns

VII. Self-Concept

-Lack of a continuous, predictable sense of self

-Poor sense of separateness

-Disturbances of body image

-Low self-esteem

-Shame and guilt

The fundamental insecure attachment mechanism is an insensitive, unpredictable or intrusively hyper-protective parenting (Ainsworth et al., 1978). Young children are at serious risk of insecurity if they read fear and dissociation in the eyes of their parents (Carlson, 1998). Trauma deprives parents of their resources and diverts their attention from the child. Few studies have empirically evaluated the different roles that family members have to show psychological suffering and resources, taking care of each other and managing the trauma. For example, if the mother shows high levels of psychological distress, the older children have low levels of discomfort or conversely, and if the mother was highly resilient the father reported low levels of resilience or vice versa.

\section{Trauma and Dissociation}

Trauma and dissociation in psychopathology are closely associated concepts, not only in the evidence of epidemiological causal relationships between development and traumatic symptoms or dissociative disorders, but also and especially in the pathogenic mechanism of trauma. Trauma activates archaic defense mechanisms of environmental threats and reactions causing detachment from the usual experience of self and the outside world and consequent dissociative symptoms (depersonalization and derealization). This gap implies an abrupt suspension of the exercise of the normal capacity for reflection and mentalizing (metacognition), and therefore an obstacle to the integration of the traumatic event in the continuity of psychic life. The disintegration of traumatic memories causes the fragmentation of representations of the self, or rather the non-integrated 
multiplicity of ego states, characterizing the pathological dissociation.

According to Jackson, the mind, which is rooted in the natural world, consists of a hierarchical organization of structures and mental functions which reflect evolutionary history, and integrate increasingly complex anatomical and functional levels in coordination between them. Each higher level coordinates with the lower ones and modulates them, building their representations. The lower level includes the simplest and automatic functions like reflections, simple automatic reactions and perceptions. Life functions belong at a higher level which integrate with the functions of the lower level by organizing them and enslaving them to increasingly complex social purposes determined by the history of the evolution of species. At the highest level the mind represents itself by integrating the activities of its bottom components. Representing itself the mind produces what we call consciousness, which for Jackson is therefore the highest level of integrative capacity of the mind, but also, for its complexity and its recent origin in the history of evolution, the most vulnerable. Janet described in detail the fragmenting and disorganizing effects of traumatic experiences on the individual's emotional and cognitive development (van der Kolk \& van der Hart, 1989). The general theory of Janet on mental functioning derives substantially from Jackson's work. Janet, like Jackson, placed the organization of mental consciousness at the summit, to which he attributed some specific integration functions. In particular, Janet considered three aspects of the integrating function of consciousness: a) the personal synthesis, which is the ability to create coherent organization of the memories and the experience of self which allows you to experience a feeling of constant identity; b) the present, which is namely the mind's active ability to focus on the present without wandering among the memories of the past, and confusing them with the present moment; c) the reality function, which consists in the ability of the mind to act according to reality, modifying it consciously according to the individual's purposes. For Janet the reality function implies a deep awareness of one's own moods and beliefs and those of others. Moreover, it expresses the free capacity of the individual to self-determination. There are many similarities between the reality function and metacognitive functions or mentalizing of contemporary literature (van der Hart et al., 2011).

In a series of controlled research on the behavior of individuals placed in a state of hypnosis, Hilgard observed that during hypnosis, trance subjects were not reacting to painful stimuli, on the other hand he showed awareness through automatic writing (form of communication sent during the state of trance, where the subject describes his experiences by writing while keeping a state of altered consciousness).

\section{Dissociative Detachment Symptoms}

April 21, 1992, in San Quentin Prison, California, Robert Harris was executed for the brutal murder of two teenagers. In 1994, in the American Journal of Psychiatry, three psychiatrists at Stanford University explored the psychological effect of the macabre events of the 18 journalists who had directly assisted him (Freinkel et al., 1994). After about one month, some questionnaires were administered to reporters. It turned out that more than half of them remembered, that at the time they had felt estranged or detached from other people, that things around them seemed unreal and "like a dream", 
and they had lost all sense of time. In addition, a third of them had perceived themselves as an outsider, or had felt detached from their thoughts, emotions and body sensations. One third had experienced mental confusion and disorientation in time and space. Despite significant dissociative symptoms from detachment that accompanied the experience, some weeks after, none of the journalists who had completed the study showed symptoms or signs of significant psychological disorders.

Researchers at Stanford University explained the intense dissociative reaction of journalists using the theses put forward by Janet. An event that evokes vehement emotions had caused the disconnection of normally stacked and integrated functional levels of mental functions. Janet's thesis does not see a mental defense against the pain of the trauma in dissociation, but a structural failure of brain functions in their hierarchical system, and this view is now also shared by some analysts. Meares, for example, states that dissociation is the manifestation of a subtle disruption of brain function engendered by the disruptive effect of the emotions associated with the traumatic event. It is not a defense on this occasion (Meares, 1999).

Experiences of depersonalization such as those described in the article by Freinkel et al. (1994), exemplify the possibility that dissociation is episodically and temporarily manifested in response to a traumatic event, without causing lasting disorders such as Post Traumatic Stress Disorder (PTSD). At times the feeling of being detached from one's emotions, or having an emotional blunting condition, which in Anglo-Saxon terminology is known as emotional numbing (Holmes et al., 2005), can persist for months after the trauma and become part of the clinical picture of acute or chronic PTSD. This state of emotional anesthesia can range from relatively bearable forms that patients describe as feeling "far from things" or "not very emotionally involved", up to extreme forms that can be experienced with a sense of total annihilation of inner life and death.

\section{Derealization}

The symptoms of detachment are often a combination the two elements of alienation from the experience of self (depersonalization) and alienation from the experience of external reality (derealization), therefore it may be useful to describe them separately. The state of derealization is the feeling of being separated from the outside world like a diaphragm, or as if it is seen through a glass plate, a metaphor that alludes to the impression that what is perceived has become untouchable (touch is the most important way in deciding that a perception is illusory). In some cases, patients choose expressions that most directly allude to a feeling of unreality that pervades not to their relationship with the outside world (that they have lost the usual intimate contact with), but the very nature of it, has become flat, colorless, lifeless, and cloaked in a veneer of unreality like things and people in a dream (Brown, 2006).

\section{Therapeutic Relationships and Overall Treatment Plans}

Currently there is no evidence that drug treatments are available for the treatment of patients with disorders resulting in the traumatic development. Experts affirm that individual psychotherapy is necessary, with the support of group therapy, and drug therapy to manage more disturbing symptoms. Individual psychotherapy of disorders re- 
sulting in traumatic development has a general problem, in addition to some specific difficulties; the symptoms that are targeted (alterations of memory and consciousness, meta-cognitive deficit, the difficulty in regulating emotions and interpersonal relationships, pathogenic beliefs of mistrust and inability to help) are simultaneously the greatest obstacles to an adequate care relationship.

\section{Care Plan}

The specificity and complexity of the treatment of disorders resulting from traumatic development has prompted the specialists of different orientations to formulate guidelines and treatment protocols to guide clinical work. Despite a few differences, there are many common aspects among the different guidelines. In particular, the common denominator of all consists in proceeding in three phases, each preliminary to the next: 1) the security stage in the stabilization of the symptoms; 2) the stage of integration of traumatic memories and the components of themselves which are dissociated; 3 ) the phase of stabilization and growth of the skills acquired (Classen et al., 2006).

In the first phase the main task of the therapy is to obtain secure conditions for the patient, both within the therapeutic relationship (by building a good alliance), and outside of the therapy with the stabilization of the most disabling symptoms (symptoms of dissociative detachment, impulsive actions and risky behavior, unruly emotions of anger, anxiety and sadness, and repetition of abusive relationship. It is necessary to achieve the goal of the first phase, the secure conditions (and a stable therapeutic alliance), to address the work of the second phase of therapy: the reconstruction and integration of traumatic memories and the beginning of the process of integration of the different parts of themselves which are dissociated. If one proceeds to the reconstruction of traumatic memories without having gained the necessary security, the patient might experience useless and counterproductive pain trauma again, and would face more frequent and severe dissociative processes, and the therapy would have an iatrogenic effect. In the third phase of the therapy, which may be faced if the first two are successful, the patient, thanks to the new sense of security and trust (in relation to self and others), the reduction of symptoms and the integration work (personal synthesis), may be assisted in new experiences which enable them to try new relationship skills with autonomy and head towards their own existential purposes.

\section{Cognitive-Behavioral Therapy of Trauma and Dissociation}

The Cognitive-Behavioral Therapies (TCC) are currently effective tools for the treatment not only of PTSD, but also DPTSc (Cloitre et al., 2010). Studies suggest the usefulness for the care of DPTSc, and the characteristic attitude of all forms of TCC, thereby focusing on the treatment directly and primarily on the symptoms. In the stabilization phase it is important to mitigate both the complex trauma symptoms (dissociation, somatization, unsettled emotions, traumatic memories, relationship difficulties, behavioral problems and dysfunctional beliefs about themselves) and those associated such as depression, anxiety, obsessive compulsive patterns, panic, abnormal eating behavior or the use of substances. Over the course of time different forms of TCC have also been specifically developed for the care of traumatic events, trauma and dissociation of the 
complex:

\section{a) Collaborative empiricism}

Unlike what happens in other forms of psychotherapy, the relationship between therapist and patient in the various forms of TCC is based on collaborative empiricism, or of a joint relationship in the pursuit of agreed goals. This type of therapeutic relationship, facilitates the attachment modulation and activation of the cooperative motivational system, plays a vital role in the treatment of all disorders resulting from traumatic development.

b) Psycho-education on the symptoms and their causes

A distinctive feature of the TCC is to provide the patient with a clear explanation about the nature of one's disturbances and the treatment processes. Psycho-education is particularly effective in reducing the suffering of patients with traumatic stories as they can often minimize feelings of guilt and shame which originate from holding themselves to be the cause of their suffering, and to begin to critically examine negative bias on themselves. In addition, the appropriate knowledge of the mechanisms of some symptoms, in particular those dissociative, allows the patients to identify them and manage them.

\section{c) Establishing targets}

Another typical aspect of the TCC is to define, prior to and during therapy, agreed and defined goals. The work for individuals and defined objectives allows, at the time of their achievement even partially, an increased sense of personal effectiveness and selfcontrol.

\section{d) Exploration of pathogenic beliefs and guided discovery}

The TCC has evolved with the purpose of modifying the beliefs that patients develop in childhood to adapt to an environment pathogen, but which then become maladaptive in other interpersonal contexts (Linehan et al., 1993).

\section{e) Exposure techniques}

In the treatment of complex trauma, the value of the corrective emotional experiences and techniques of gradual exposure to feared situations cannot be underestimated. The exposure techniques can take place in vivo or in driven imagination, which is widely used in the TCC and its derivations (such as EMDR) for the treatment of traumatic memories (Rauch \& Foa, 2006).

\section{f) Possibility of integration with other treatments}

The TCC, traditionally, does not exclude the simultaneous use of other therapeutic techniques but rather encourages it. It also lends itself well to be combined with either drug therapies or with both interventions integrated multiple settings.

\section{Group, Family and Couples Therapy}

Since the definition of PTSD, treatment approaches have been developed to address the main problems of the dissociative traumatic dimension in group therapy or support groups. Trauma and its memories, symptoms of hyperactivity or emotional blunting, unruly emotions, dissociative symptoms, relationship difficulties, risk behaviors (Bloom, 2000). In recent years therapeutic techniques specific to the systemic-relational DPTSc have been improved (Courtois \& Ford, 2009). Group, family and couples therapy are 
increasingly being used in Integrated Multi-setting Treatments (TMSI) of difficult patients (Farina \& Rainone, 2005). The use of therapeutic, support and self-help groups appears to be important especially in the first phase of the treatment protocol. In these groups, the patients can receive information about their symptoms and compare their experiences on a parity basis with others. Therefore, according to the theory that attributes to the joint cooperative relationship, which is the highest level of usability of metacognitive capacity, they can begin to reflect about themselves better than they would in the initial individual dialogue contexts with a therapist perceived in the motivational dimension of attachment (such as a potential source of care and comfort) or rank (such as a dominant figure who gives prescriptions to which one must comply). In addition, those in group contexts who suffer from similar disorders counteract the sense of isolation and alienation which is one of the most widespread feelings in DPTSc. Family therapy meetings in contexts of co-therapy with individual treatment (TMSI) can prove crucial during the early stages of the treatment of patients who are still very young and live in neglectful families with emotionally fragile parents who are vulnerable or otherwise violent, and whose abusive behavior in the past formed the roots of the DPTSc. The goal of family intervention in these cases is to mitigate the intense emotional atmosphere, so that individual care can take place with the least of possible interference on the part of family members.

\section{Drug Therapies in the Development of Traumatic Treatment}

The complexity of adaptation to early relational trauma does not allow the management of disorders that derive exclusively with psychotropic drugs (TPF). However, it often happens that in DBP, in PTSD and dissociative disorders, the use of drugs in patients with DPTSc is indicated for the symptomatic management of some specific areas (such as emotional dysregulation or poor impulse control, irritability and intrusive symptoms) or to treat frequent comorbid disorders such as mood or anxiety. Although the lack of recognition of DPTSc nosographic as an autonomous entity has hampered the realization of specific research protocols, several studies have examined the use of TPF in patients with complex trauma, maltreatment and child abuse and concluded that the use of psychotropic drugs is indicated as an aid in psychotherapy and never as exclusive treatment (van der Kolk, 2005). In these patients, TPF has the main purpose of stabilizing the patient in the first phase of the therapy and of allowing the therapist to proceed in the realization of the first faces of the treatment; for example, therapeutic alliance building, the research of patient security, and the modulation of the most disabling symptoms. For this purpose the use of antidepressant medications, mood stabilizers and atypical antipsychotics has proved useful to regulate unruly emotions, irritability and dysphoria, to contain the disturbances of behavior and self-aggressive and suicidal acts, and to mitigate somatization. The categories of drugs used for this purpose are the selective serotonin reuptake inhibitors (SSRIs), atypical antidepressants, beta-blockers, mood stabilizers, and second-generation antipsychotics. Patients with DPTSc are often turning to clinicians due to the emergence of an anxiety disorder (panic attacks, generalized anxiety, claustrophobia), an alteration of mood, substance abuse, eating disorders or the onset of a disorder related to or associated with complex trauma. In these 
cases it is often necessary to resort to TPF to overcome the initial symptoms that otherwise would inhibit the general therapeutic protocol.

TPF therapies often have management problems. One of these is that patients with DPTSc often have a low adherence to the therapeutic rules and therefore also poor compliance to TPF. Furthermore, the frequent impulsiveness problems require particular caution in prescribing psychotropic substances, of which the patient may abuse, and in extreme cases, even assume excessive doses for suicidal acts.

\section{Conclusion}

Studies and knowledge about the consequences of traumatic events on mental health among children and adolescents are increasing steadily. Aspects of development are informative for understanding the diseases related to trauma, dissociation, and derealization and to their recovery, whether spontaneous or after a treatment. Trauma is not a healthy presence in the field of human development. Children need all their resources to pursue social regulations and cognitive and emotional development tasks, therefore, the processing of traumatic events is a very difficult task for the psyche of a child. Traumatic events often involve a vicious circle in the development. Children, when they are traumatized, are in urgent need of their socio-emotional resources to survive because the trauma is testing these same resources, such as the intimate relations of friendship and the narrative ability to understand and integrate the trauma. Traumatic events are transmitted to children by parents and other family members, so understanding the interactive relationships, patterns of attachment, behavioral and family systems is particularly important during treatment.

\section{References}

Ainsworth, M., Waters, E., \& Wall, S. (1978). Patterns of Attachment. A Psychological Study of the Strange Situation. Hillslade, New Jersey: Lawrence Eribaum Associates.

Almqvist, K., \& Broberg A. G. (1997). Silence and Survival: Working with Strategies of Denial in Families of Traumatized Pre-School Children. Journal of Child Psychotherapy, 23, 417-435. https://doi.org/10.1080/00754179708254560

Bloom, S. (2000). In International Handbook of Human Response to Trauma. New York: Kluwen Academic/Plenum Press.

Brown, R. J. (2006). Different Types of Dissociation Have Different Psychological Mechanisms. Trauma Dissociation, 7, 7-28. https://doi.org/10.1300/J229v07n04_02

Carlson, E. A. (1998). A Prospective Longitudinal Study of Attachment Disorganization/Disorientation. Child Development, 69, 1107-1128. https://doi.org/10.1111/j.1467-8624.1998.tb06163.x

Classen, C. C., Pain, C., Field, N. P., \& Woods, P. (2006). Post-Traumatic Personality Disorder: A Reformulation of Complex Posttraumatic Stress Disorder and Borderline Personality Disorder. Psychiatric Clinics of North America, 29, 87-112. https://doi.org/10.1016/j.psc.2005.11.001

Cloitre, M., Stovall-McClough, K. C., Nooner, K., Zorbas, P., Cherry, S., Jackson, C. L. et al. (2010). Dimensione dissociativa e trauma dello sviluppo. Treatment for PTSD Related to Childhood Abuse: A Randomized Controlled Trial. American Journal of Psychiatry, 167, 915-924. https://doi.org/10.1176/appi.ajp.2010.09081247

Collishaw, S., Dunn, J., \& O’Connor, T. G. (2007). A Longitudinal Study of Parents and Children Study Team Maternal Childhood Abuse and Offspring Adjustment over Time. Developmental 
Psychopathology, 19, 367-383. https://doi.org/10.1017/S0954579407070186

Courtois, C. A., \& Ford, J. D., (2009). Treating Complex Traumatic Stress Disorders (Adults) Scientific Foundations and Therapeutic Models (Edited by C. A. Courtois, \& J. D. Ford).

Farina, B., \& Rainone, A. (2005). Una pratica senza teoria. Storia delle psicoterapie a setting multipli per i pazienti gravi. In G. Liotti, B. Farina e A. Rainone (Eds.), Due terapeuti per un paziente. Roma: Laterza.

Feeny, N. C., Foa, E. B., Treadwell, K. R. H., \& March, J. (2004). Post-Traumatic Stress Disorder in Youth: A Critical Review of the Cognitive and Behavioral Treatment Outcome Literature. Professional Psychology: Research and Practice, 35, 466-476. https://doi.org/10.1037/0735-7028.35.5.466

Freinkel, A., Koopman, C., \& Spiegel, D. (1994). Dissociative Symptoms in Media Eyewitnesses of an Execution. American Journal of Psychiatry, 151, 1335-1339. https://doi.org/10.1176/ajp.151.9.1335

Hodges, E. V., Boivin, M., Vitaro, F., \& Bukowski, W. M. (1999). The Power of Friendship: Protection against an Escalating Cycle of Peer Victimization, Developmental Psychology, 35, 94101. https://doi.org/10.1037/0012-1649.35.1.94

Holmes, E. A., Brown, R. J., Mansell, W., Fearon, R. P., Hunter, E. C., Frasquilho, F., \& Oakley, D. A. (2005). Are There Two Qualitatively Distinct Forms of Dissociation? A Review and Some Clinical Implications. Clinical Psychology Review, 25, 1-23. https://doi.org/10.1016/j.cpr.2004.08.006

Howe, M. L., Cicchetti, D., Toth, S. L., \& Cerrito, B. M. (2004). True and False Memories in Maltreated Children. Child Development, 75, 1402-1417. https://doi.org/10.1111/j.1467-8624.2004.00748.x

Janoff-Bulman, R. (1997). Understanding Reactions to Traumatic Life Events. The Harvard Mental Health Newsletter, 14.

Leavitt, K. S., Gardner, S. A., Gallagher, M. M., \& Schamess, G. (1998). Severely Traumatized Siblings: A Treatment Strategy. Clinical Social Work Journal, 26, 16-23. https://doi.org/10.1023/A:1022897512333

Linehan, M. M., Heard, H. L., \& Armstrong, H. E. (1993). Naturalistic Follow-Up of a Behavioral Treatment of Chronically Para-Suicidal Borderline Patients. Archives of General Psychiatry, 50, 971-974. https://doi.org/10.1001/archpsyc.1993.01820240055007

Maughan, A., \& Cicchetti, D. (2002). Impact of Child Maltreatment and Inter-Adult Violence on Children's Emotion Regulation Abilities and Socio-Emotional Adjustment. Child Development, 73, 1525-1542. https://doi.org/10.1111/1467-8624.00488

Meares, R. (1999). The Contribution of Hughlings Jackson to an Understanding of Dissociation. American Journal of Psychiatry, 156, 1850-1855.

Moradi, A. R., Neshat Doost, H. T., Taghavi, M. R., Yule, W., \& Dalgleish, T. (1999). Everyday Memory Deficits in Children and Adolescents with PTSD: Performance on the Rivermead Behavioural Memory. Journal of Child Psychological Psychiatry, 40, 357-361. https://doi.org/10.1111/1469-7610.00453

O'Brien, S. F., \& Bierman, K. L. (1988). Conceptions and Perceived Influence of Peer Groups: Interviews with Preadolescents and Adolescents. Child Development, 59, 1360-1365. https://doi.org/10.2307/1130498

Ozer, E. J., Best, S. R., Lipsey, T. L., \& Weiss, D. S. (2004). Predictors of Post-Traumatic Stress Disorder and Symptoms in Adults. Psychological Bulletin, 129, 52-73. https://doi.org/10.1037/0033-2909.129.1.52

Pynoos, R. S., Steinberg, A. M., \& Piacentini, J. C. (1999). A Developmental Psychopathology Model of Childhood Traumatic Stress and Intersection with Anxiety Disorders. BiologicalPsychiatry, 46, 1542-1554. https://doi.org/10.1016/s0006-3223(99)00262-0 
Rauch, S. A. M., \& Foa, E. B. (2006). Emotional Processing Theory and Exposure Therapy for PTSD. Journal of Contemporary Psychotherapy, 36, 61-67. https://doi.org/10.1007/s10879-006-9008-y

Scheeringa, M., \& Zeanah, C. H. (2001). A Relational Perspective on PTSD in Early Childhood. Journal of Traumatic Stress, 14, 799-815. https://doi.org/10.1023/A:1013002507972

Shields, A., \& Cicchetti, D. (1998). Reactive Aggression among Maltreated Children: The Contributions of Attention and Emotion Dysregulation. Journal of Clinical Child Psychology, 27, 381-395. https://doi.org/10.1207/s15374424jccp2704_2

Tremblay, R. E. (2000). The Development of Aggressive Behavior during Childhood: What Have We Learned in the Past. Journal of Behavioral Development, 24, 129-141. https://doi.org/10.1080/016502500383232

van der Hart, O., Nijenhuis, E. R. S., \& Steele, K. (2011). Fantasmi nel sé. Trauma e trattamento della dissociazione strutturale. Milano: Raffaello Cortina.

van der Kolk, B. A. (1997). The Psychobiology of Posttraumatic Stress Disorder. Journal of Clinical Psychiatry, 58, 16-24.

van der Kolk, B. A. (2005). Developmental Trauma Disorder: Toward a Rational Diagnosis for Children with Complex Trauma Histories. Psychiatric Annals, 35, 401-408.

van der Kolk, B. A., \& van der Hart, O. (1989). Pierre Janet and the Breakdown of Adaptation in Psychological Trauma. American Journal of Psychiatry, 146, 1530-1540.

https://doi.org/10.1176/ajp.146.12.1530

\section{Submit or recommend next manuscript to SCIRP and we will provide best service} for you:

Accepting pre-submission inquiries through Email, Facebook, LinkedIn, Twitter, etc. A wide selection of journals (inclusive of 9 subjects, more than 200 journals)

Providing 24-hour high-quality service

User-friendly online submission system

Fair and swift peer-review system

Efficient typesetting and proofreading procedure

Display of the result of downloads and visits, as well as the number of cited articles

Maximum dissemination of your research work

Submit your manuscript at: http://papersubmission.scirp.org/

Or contact sm@scirp.org 